\title{
Post-operative Rehabilitation for Scapular Muscle Reattachment: A Case Report
}

\author{
Wendy Burke, PT, DPT, MS, OCS, CCRC ${ }^{1}$, W Ben Kibler, MD², Tim L Uhl, PhD, PT, ATC ${ }^{3}$ \\ ${ }^{1}$ Department of Biokinesiology and Physical Therapy, University of Southern California, ${ }^{2}$ Shoulder Center of Kentucky, ${ }^{3}$ Department of Physical \\ Therapy, University of Kentucky \\ Keywords: rehabilitation, scapular dyskinesis, shoulder pain, therapeutic exercise, movement system \\ https://doi.org/10.26603/001c.21240
}

\section{International Journal of Sports Physical Therapy}

Vol. 16, Issue 2, 2021

\section{Background and Purpose}

Scapular muscle detachment is a rare orthopedic problem that has been described in the literature in patients following traumatic events involving traction, direct trauma, or a motor vehicle accident. The purpose of this case report is to describe the post-operative rehabilitation following scapular muscle reattachment surgery. Unique to this case report is the patient's perspective, an orthopedic physical therapist with 25 years of experience.

\section{Case Description}

A 47-year-old female physical therapist experienced a traction injury to bilateral upper extremities during a medical procedure resulting in bilateral rhomboid, and bilateral lower trapezius muscles were detached from the medial scapular border. Reconstruction of the left scapulothoracic musculature occurred five and one-half years post-injury with the right repaired one year later. This case report describes the rehabilitation program that took one-year to recover for each arm with a period of protected motion for 16-weeks and gradual return to function as a manual physical therapist over a period of one-year.

\section{Outcomes}

The American Shoulder and Elbow Surgeons (ASES) Standardized Assessment Form and pain-free range of motion was used pre- and postoperatively. Left and right shoulder pre-operative ASES scores were 68 and 72, respectively. At the one-year post-operative the left shoulder was rated at 82 and the right shoulder was 90 . Pain-free range of motion was achieved in both arms by one year. Functional limitations requiring strength overhead were the slowest to return and were not completely back at one year following either surgery.

\section{Discussion}

Rehabilitation protocols for scapular muscle reattachment surgery are not commonly available to allow physical therapists to guide their patients and structure a rehabilitation program. This case report provides a sample pre-operative set of educational guidelines and a post-operative protocol for use after scapular reattachment surgery. This case report is unique because it offers a patient perspective who is a physical therapist and underwent this surgery twice. Therefore, providing insight on how to prepare for such a unique operation. The slow recovery is due to three issues 1) the prolonged time from injury to diagnosis created significant muscle wasting and muscular imbalance of surrounding tissues, 2) once this tissue was repaired it requires months of protection to recover, 3 ) the involved scapulothoracic muscle have to regain adequate strength as the foundation for upper extremity functions.

\footnotetext{
a Corresponding Author:

Wendy Burke

Department of Biokinesiology and Physical Therapy

University of Southern California, Los Angeles, CA, USA

Wendyburke01@gmail.com
} 


\section{Level of evidence}

Level 5

\section{BACKGROUND}

Abnormal scapular position or movement, called scapular dyskinesis, is commonly seen in patients with shoulder injury or pain. ${ }^{1-7}$ There are multiple causes of scapular dyskinesis including bony (e.g. fracture), neurologic (e.g. long thoracic or accessory nerve palsy), and muscular dysfunction (e.g. soft tissue inflexibility, muscle weakness, inhibition, or imbalance). 1,5,6,8,9 The majority of scapular dyskinesis seen by the therapist is the result of muscular imbalances. Treatments directed at restoring muscular length, strength, and motor control are effective when applying good clinical reasoning to the biomechanics of the upper quadrant. ${ }^{1-6,10-13}$ However, there is a subset of patients presenting with scapular dyskinesis, that have chronic pain and have not responded to logical and appropriate rehabilitation that have been found to have a periscapular muscular detachment. ${ }^{3}$ Patients with a scapular muscle detachment can obtain pain reduction and functional improvement by surgically reattaching the muscles first, then rehabilitation. ${ }^{3}$ To date, there is little information regarding the post-surgical rehabilitation of periscapular muscle tears in the literature.

This case report provides guidelines and insight for rehabilitation after surgical scapular muscle reattachment as well as how this rehabilitation program differs from the more typical rotator cuff repair, which also involves reattachment of the musculotendinous structures. The purpose of this case report is to describe the post-operative rehabilitation following scapular muscle reattachment surgery. This case report is unique as an experienced physical therapist underwent the surgical procedures and was able to provide a perspective of rehabilitation from the combined patient and physical therapist perspective. The subject of the study has been informed that the data and photos taken would be submitted for publication and has given consent.

\section{CASE DESCRIPTION}

A 47-year-old female physical therapist sustained a traction injury to bilateral scapulothoracic muscles during a medical procedure. The mechanism of injury occurred while the subject was awaiting anesthesia in the operating room. Both upper extremities were strapped and restrained on a board with the left arm abducted at $100^{0}$ and the right arm abducted at $80^{\circ}$ with elbows extended. A nurse grabbed both ankles and pulled forcefully to move the subject down to the end of the gurney causing a bilateral traction event to the scapulothoracic musculature. The subject recalled immediately feeling a sharp pain with severe burning in bilateral periscapular musculature. Soon after notifying the surgical staff, anesthesia was administered, and the intended procedure performed. Upon awaking from anesthesia, the subject reported significant pain in the periscapular region, neck, left arm and difficulty breathing. These symptoms were attributed to a pneumothorax, confirmed by radiographs, and the patient was immediately returned to surgery for insertion of a chest tube and discharged to home that same day with chest tube removed thirteen days later and return to work as a physical therapist two days after insertion of the chest tube.

From the initial incident, symptoms of periscapular muscle burning, rib pain, cervical pain, and increasing weakness with arm motions overhead and away from the body persisted. Conservative physical therapy interventions along with self-management consisted of rib mobilizations, scapular taping, wearing a postural support shirt, ultrasound to the glenohumeral joint, anti-inflammatory medications, and modalities such as ice and heat daily. Strengthening exercise consisted of machine exercises in the gym (such as leg press, abdominal crunches, latissimus pull downs, shoulder press, chest press, triceps and biceps), light free weights and elastic resistance exercises for the upper extremities including specific scapular exercises for adduction, depression, and elevation. Treadmill and bicycle exercises were incorporated to replace running for cardiovascular fitness. These activities were continued for approximately four years.

Despite consistent interventions there was a significant and progressive decrease in strength and range of motion in both shoulders along with an increase in periscapular, cervical, and shoulder pain. Pre-injury fitness activities including running or bicycling had to be eliminated secondary to increased bilateral shoulder and arm pain. The shoulder pain and periscapular burning disrupted sleep every two to three hours. The subject, as a therapist, felt as though she failed at doing what she commonly instructed her patients to perform daily. And, assuming that there was no frank nerve injury, that it had to be a motor control or muscle length - muscle strength balancing problem; thus, within her skill set to resolve. The dosage of exercises, repetitions, or weights were continually modified to make improvements.

Symptoms persisted and increased from local burning along the medial aspect of the scapula to constant left acromioclavicular joint pain, radiating pain down the left upper extremity to $4^{\text {th }}$ and $5^{\text {th }}$ digits, posterior shoulder pain bilaterally with a "cramping" sensation on the bilateral posterior lateral scapula, cervical pain, headaches, and a sensation of arm feeling very heavy that needed to be supported to mitigate symptoms. The subject's job as a physical therapist was compromised requiring constant modifications of her body mechanics due to the inability to lift, carry, push or pull the weight of her own extremity, or that of her patient's. The only relief of symptoms was found in sitting with the medial scapular borders compressed onto the trunk with the back of a firm chair and the left upper extremity supported in abduction and slight external rotation. This maneuver of manual compression the scapula onto the trunk for stability mimics the "scapular stabilization" test performed in the clinic. Obtaining such significant and immediate relief confirmed to the therapist/subject that the primary cause of the problem has to do with the lack of scapular stability.

Four years after the initial injury, the subject sought an 
orthopedic consult secondary to constant left acromioclavicular joint (ACJ) pain and increasing difficulty with all functional activities. An injection to the left ACJ provided immediate relief for a couple days and diminished to $50 \%$ in the AC for two weeks but no meaningful change in symptoms to the rest of the shoulder or arm. A subsequent arthrogram revealed a tear in the upper labrum which involved the biceps tendon anchor. The arthrogram was negative for posterior labral tear and rotator cuff tear. A bicipital tenodesis was proposed but declined by the subject.

Electrodiagnostic testing was ordered to rule out nerve injury. There was no electromyographic evidence of cervical radiculopathy, plexopathy, dorsal scapular neuropathy, long thoracic nerve palsy, spinal accessory neuropathy or peripheral neuropathy on the left musculature. All nerve conduction velocities for median and ulnar nerves were greater than $53 \mathrm{~m} / \mathrm{s}$ ruling out peripheral neuropathy. Antidromic sensory nerve conduction velocities were $36-38 \mathrm{~m} /$ $\mathrm{s}$ for radial, ulnar, and median nerves which are within normal values for sensory nerve testing. Innervation to the muscles were intact yet strengthening and motor control exercises were not effective and injections into the joint provided no relief. The subject suspected a scapular muscle detachment as a possible cause of the chronic symptoms. The subject sought out another orthopedic surgeon who specializes in scapular muscle dysfunction.

\section{SCAPULAR SURGEON CLINICAL EXAMINATION}

A thorough clinical examination was carried out by the orthopedic surgeon. (Table 1) The clinical examination revealed that there was significant scapular muscle involvement. Based on the failure of injections, rehabilitation, negative EMG, and the duration of symptoms the clinical examination was consistent with a diagnosis of scapular muscle detachment injury bilaterally. ${ }^{3}$ Surgery for reattachment of the left scapular muscles, which were more involved was discussed with the subject, along with the postoperative rehabilitation limitations. The patient consented and was scheduled for surgery 5.5 years from initial injury. This time frame for diagnosis and relevant surgery is consistent with reported literature of an average of four years. ${ }^{3}$ The right scapula musculature was repaired approximately one year later.

\section{SCAPULAR MUSCLE REPAIR SURGERY}

The goals of scapular muscle repair surgery are to determine the extent of the muscle damage to the periscapular muscles, identify and mobilize the damaged tissue, and reestablish normal attachment of the muscles to the bone. For this subject, the left scapula required reattachment of the middle and lower trapezius and the rhomboid major muscles. The right scapular muscle reattachment was performed 11 months after the left shoulder. This surgery required reattachment of the lower trapezius, rhomboid major and minor. Detailed description of this surgical procedure has been previously described. ${ }^{3}$

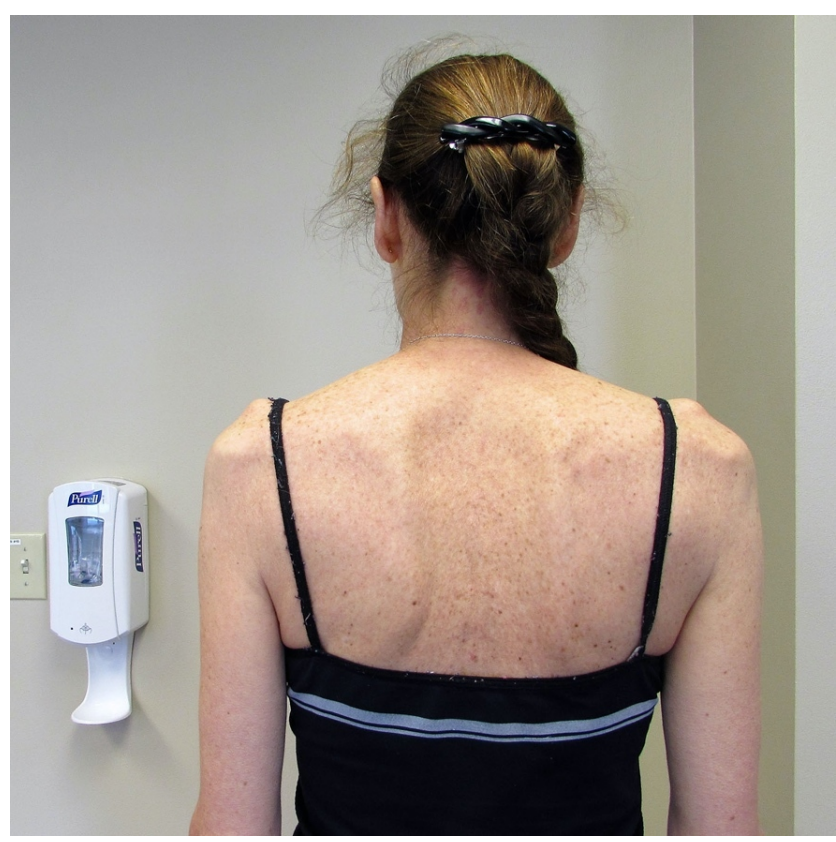

Figure 1: Pre-operative scapular position.

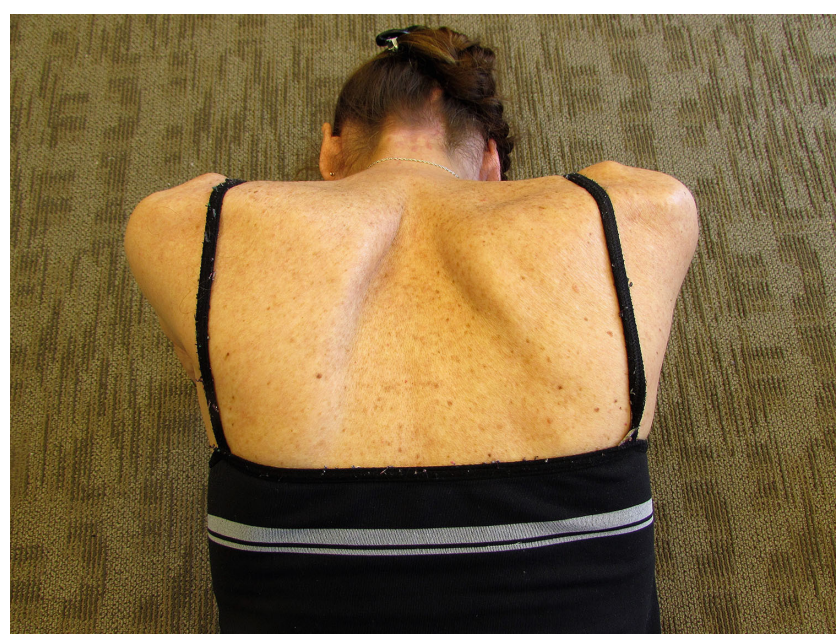

Figure 2: Preoperative closed chain function of scapulae

\section{POST OPERATIVE REHABILITATION}

Post-operative management of scapular muscle reattachment is outlined in a protocol available at the Lexington Clinic web site: https://www.lexingtonclinic.com/assets/ files/Documents/2018 LC_Orthopedices_Scapular_Mus-

cle_Reattachment_2.pdf (Copy and paste the link into your web browser to access the protocol) These guidelines are modified for each patient based on the surgeon's assessment of tissue quality, size of tear, and individual patient's health. A significant modification that is not described in the Lexington Clinic program relates to pre-operative education. As this subject underwent the surgery twice, development of a pre-operative program made recovery much easier following the second surgery. Pre-operative treat- 
Table 1: Scapular surgeon's clinical examination

Observation Static scapular position: excessive scapular internal rotation causing winging of the medial border and protraction bilaterally (Figure 1).

Dynamic scapular mobility: positive scapular dyskinesis during active elevation in all planes of motion.

Palpation A palpable divot was evident along the superior medial border of the scapula where the subject described the "burning" sensations on both scapulae $(L>R)$. Palpation was performed by lightly sliding his hand along the medial border of the scapula from superior to inferior. This was a different technique than previously performed by other surgeons where the palpation was only a deep pressure applied by the thumb from posterior to anterior.

Tenderness and increased tone were noted in the pectoralis minor, infraspinatus, latissimus dorsi, teres major and minor muscles bilaterally.

\begin{tabular}{|c|c|c|c|c|c|c|}
\hline \multirow{7}{*}{$\begin{array}{l}\text { Range of } \\
\text { Motion }\end{array}$} & & \multicolumn{2}{|l|}{ Left } & \multicolumn{3}{|l|}{ Right } \\
\hline & $\begin{array}{l}\text { Elevation with scapular } \\
\text { dyskinesis }\end{array}$ & \multicolumn{2}{|l|}{$100^{0}$} & \multicolumn{3}{|l|}{$120^{0}$} \\
\hline & Hand Behind Back & \multicolumn{2}{|l|}{ T6 } & \multicolumn{3}{|l|}{ T10 } \\
\hline & $\begin{array}{l}\text { Active External } \\
\text { Rotation at side }\end{array}$ & \multicolumn{2}{|l|}{$30^{0}$} & \multicolumn{3}{|l|}{$45^{0}$} \\
\hline & $\begin{array}{l}\text { Passive External } \\
\text { Rotation at side }\end{array}$ & \multicolumn{2}{|l|}{$60^{0}$} & \multicolumn{3}{|l|}{$60^{\circ}$} \\
\hline & $\begin{array}{l}\text { Active External } \\
\text { Rotation at } 90^{0^{*}}\end{array}$ & $45^{0}$ & & \multicolumn{3}{|l|}{$45^{0}$} \\
\hline & \multicolumn{6}{|c|}{ *Limited by weakness not by pain } \\
\hline \multirow[t]{2}{*}{$\begin{array}{l}\text { Special } \\
\text { Tests }\end{array}$} & \multirow{2}{*}{\multicolumn{6}{|c|}{$\begin{array}{l}\text { Scapular reposition test: Positive bilaterally; indicating that scapular stability is key for rehab and lack of the } \\
\text { stability contributes to the cause of the symptoms. } \\
\text { Scapular assistance tests: Positive bilaterally; indicating that proper scapular motion is needed for symptom } \\
\text { resolution. }\end{array}$}} \\
\hline & & & & & & \\
\hline \multirow[t]{6}{*}{ Strength } & \multicolumn{6}{|c|}{$\begin{array}{l}\text { Manual muscle testing was performed manually using the grading system described by Kendall. }{ }^{14} \text { Both shoulders } \\
\text { were painful with inability to obtain or maintain scapular neutral on either extremity. A muscle contraction was } \\
\text { palpable along the medial attachment at the thoracic spine with attempts at scapular adduction and depression. } \\
\text { Upper extremity weight bearing demonstrated increased bilateral scapular winging, abduction, and upward } \\
\text { rotation as the antagonistic rhomboid musculature was not engaged. (Figure } 2 \text { ) }\end{array}$} \\
\hline & Pre-operative & \multicolumn{2}{|l|}{ Left } & \multicolumn{3}{|l|}{ Right } \\
\hline & Rhomboids & \multicolumn{2}{|l|}{$1+/ 5$} & \multicolumn{3}{|l|}{$2-/ 5$} \\
\hline & Middle Trapezius & \multicolumn{2}{|l|}{$1+/ 5$} & \multicolumn{3}{|l|}{$2-/ 5$} \\
\hline & Lower Trapezius & \multicolumn{2}{|l|}{$1+/ 5$} & \multicolumn{3}{|l|}{$2-/ 5$} \\
\hline & $\begin{array}{l}\text { Serratus Anterior with } \\
\text { protraction }\end{array}$ & $4 / 5$ & & $4 / 5$ & & \\
\hline Outcomes & $\begin{array}{l}\text { The patient completed } t \\
\text { pre-operatively and at s } \\
\text { on level of perceived fur } \\
\text { American Shoulder and } \\
\text { right. }\end{array}$ & $\begin{array}{l}\text { nerican Sh } \\
\text { uent follov } \\
\text { with } 100 \\
\text { Surgeon's }\end{array}$ & $\begin{array}{l}\text { nd Elbow Sur } \\
\text { e points. The } \\
\text { dicating no p } \\
\text { or left and ri }\end{array}$ & $\begin{array}{l}\text { (ASES) pat } \\
\text { is based on } \\
\text { id normal fu } \\
\text { oulders. The }\end{array}$ & $\begin{array}{l}\text { reported fu} \\
\text { olevel of cu } \\
\text { on of the ur } \\
\text { was opera }\end{array}$ & $\begin{array}{l}\text { and pain scale } \\
\text { ain and } 50 \% \\
\text { remity. } 15 \\
\text { ear prior to the }\end{array}$ \\
\hline & $\begin{array}{l}\text { Left } \\
\text { Pain } \\
\text { Score }\end{array}$ & $\begin{array}{l}\text { Left } \\
\text { Function } \\
\text { Score }\end{array}$ & $\begin{array}{l}\text { Left ASES } \\
\text { Total Score }\end{array}$ & $\begin{array}{l}\text { Right } \\
\text { Pain } \\
\text { Score }\end{array}$ & $\begin{array}{l}\text { Right } \\
\text { Function } \\
\text { Score }\end{array}$ & $\begin{array}{l}\text { Right ASES } \\
\text { Total Score }\end{array}$ \\
\hline & $\begin{array}{l}\text { Pre- } \\
\text { operative }\end{array}$ & 38 & 68 & 35 & 37 & 72 \\
\hline & 3 months & 15 & 50 & $\begin{array}{l}\text { Not } \\
\text { captured }\end{array}$ & $\begin{array}{l}\text { Not } \\
\text { captured }\end{array}$ & $\begin{array}{l}\text { Not } \\
\text { captured }\end{array}$ \\
\hline & 6 months & 35 & 70 & $\begin{array}{l}\text { Not } \\
\text { captured }\end{array}$ & $\begin{array}{l}\text { Not } \\
\text { captured }\end{array}$ & $\begin{array}{l}\text { Not } \\
\text { captured }\end{array}$ \\
\hline & $\begin{array}{l}12 \\
\text { months }\end{array}$ & 42 & 82 & 43 & 47 & 90 \\
\hline
\end{tabular}

ments help the patient prepare for activities of daily living (ADL's) $s$ in the first six weeks when no formal therapy is prescribed and lessens the chance of damaging the healing tissues. (Appendix A) The rehabilitation program that was carried out for this subject follows the four-phase program described above in the protocol from the Lexington Clinic. 
Specific treatments are detailed below and in Appendix B to explain how the goals were achieved.

One critical difference that physical therapist must keep in mind is that rehabilitation of scapular muscle detachment is slower and has different precautions than the more common rehabilitation after a rotator cuff repair. With a rotator cuff repair, retraining the scapular muscles for stability is relatively easy in the first few weeks after surgery, and helps to restore normal kinematics while the repaired cuff is healing. However, for scapular reattachment surgery, the rhomboids and trapezius muscles that have been surgically reattached need to heal before retraining. Therefore, motions that cause scapular protraction or depression are contraindicated as they place tension on the repair. Because scapular stability is necessary prior to glenohumeral motion, the expectations for acquiring functional arm range of motion differ greatly between the two post-operative groups. In patients with a rotator cuff repair, passive elevation to $90^{0}$ would be expected to be obtained by six weeks and nearly full passive motion re-established by 12 weeks. ${ }^{16}$ Generally, and as in this case report, with scapular muscle reattachment, the goal was passive elevation to $90^{\circ}$ at $12-16$ weeks with good scapular control. That is nearly double the time frames compared to a rotator cuff repair. At four- and five-months post-operatively, passive elevation would approach $120^{\circ}$ and $160^{\circ}$, respectively with active range of motion trailing by approximately $20^{\circ}$. Full passive elevation with ease was not obtained until at least six months postsurgery and full active elevation along with strength recovery took approximately one year in this case.

\section{REHABILITATION PROGRAM SUMMARY}

Please refer to Appendix B that outline treatment goals, precautions, therapeutic activities allowed, symptoms associated with recovery, and manual therapy suggestions which serves as supplemental to the explicit Lexington Clinic protocol.

\section{PHASE 1: (0-6 WEEKS)}

The pre-operative patient education sessions are most helpful during this phase. The pre-operative sessions serve to help the patient perform simple ADLs such as dressing, don and doff sling, and sleeping positions. It also teaches the patient what types of activities the uninvolved extremity performs that will increase stress to the surgical side and may be harmful to the healing tissues.

\section{PHASE 2: (6-8 WEEKS)}

The second phase is when supervised physical therapy begins. However, after a period of immobilization and a surgical intervention preceded by multiple years of muscle imbalance affecting the scapulothoracic joint, obtaining a neutral position of the scapula on the rib cage can be challenging. A cascade of events contributes to difficulty in obtaining a scapular neutral position. First, the unattached muscles are overpowered by healthy antagonist muscles, (i.e., serratus anterior, latissimus dorsi, teres major, pectoralis minor and major muscles) which have been func- tioning unopposed and compensating to provide stability for several years. Additionally, the surgical trauma affecting infraspinatus followed by six weeks of immobilization decreases the fascial and muscle extensibility. When the humerus moves away from the body, such as with passive range of motion, the lack of infraspinatus muscle flexibility causes the scapula to be pulled laterally or into protraction placing tension on the repair. Therefore, restoration of normal muscle length and fascial mobility is necessary to restore the normal kinematics, with the scapula in neutral. Techniques unique to this case and found beneficial in the early phases of rehabilitation are described below and in Appendix B.

\section{STRETCHING AND SOFT TISSUE INTERVENTIONS}

The following three manual techniques were found to be effective in the clinic and progressed as a home program as subject tolerated.

1. Scapulothoracic neutral: With the patient lying on the uninvolved side the treating therapist can passively move the scapula into neutral with retraction and elevation. The therapist stands in front of the patient and places one hand on the scapula with the other over the anterior shoulder and acromioclavicular joint taking care to support the forearm to prevent rotation. The therapist passively moves the scapula through a small arc posteriorly into retraction, with slight elevation, and downwardly rotates the scapula so that the medial border of the scapula is parallel to the thoracic spine. Retraction can be obtained if the therapist aims the medial superior angle of the scapula back and up toward C7-T1. The therapist passively applies downward rotation to the scapula so the medial border is parallel to the thoracic spine. Note, downward rotation is not depression of the scapula. Avoid depression of the scapula which creates tension on the rhomboids and should be avoided it tissue is involved. In the clinic, the therapist can facilitate scapular external rotation by manually stabilizing the medial border of the scapula against the rib cage. (Figure 3) Note, the therapist cannot force this motion but merely directs it and waits until the body essentially "invites them in". This should be repeated four to six times until the scapula moves passively into retraction with little resistance. Although the position may follow a PNF direction, only the passive retraction - elevation portion is used. ${ }^{17}$ Traditional PNF techniques with a quick stretch into end ranges are not appropriate at this stage to protect the repair.

2. Side lying soft tissue massage: Once the scapula is retracted passively into a neutral position in side-lying, the therapist stabilizes the scapula to protect the repair and performs gentle soft tissue mobilization to the superficial layers of the infraspinatus, posterior deltoid, and proximal triceps. Mobilizing these posterior scapulohumeral muscles and fascia will allow the initial $20-30^{\circ}$ of humeral elevation in the frontal plane during this phase of rehabilitation. (Figure 4)

3. Pectoralis muscle stretching: Tight and shorten pectoralis muscles causes scapular anterior tilt and protraction. Therefore, increasing muscle length is a prerequisite for posterior tilt and retraction to obtain scapular neutral. The subject is in a supine position to stabilize the scapula on the 
table while the therapist applies gentle posterior pressure over the anterior-superior aspect acromion toward posterior tilt with the humerus in neutral rotation, $30^{\circ}$ of abduction, and $0-10^{\circ}$ of flexion. In hook lying the patient crosses their leg and rotates the lower extremity away from the opposite side and takes slow deep breaths to relax and stretch the pectoral muscles. (Figure 5 ) The patient should feel the muscle stretch along the muscle belly and not at the anterior humeral head or AC joint. Each stretching technique should be performed gently and slowly for 5-8 repetitions, holding each for 20-60 seconds and repeated 2-3 times a day.

\section{STRENGTHENING INTERVENTIONS}

1. Scapular retraction isometrics: Isolated sub-maximal muscle activity is necessary for normal shoulder kinematics and serves as the basis for progression of neuromotor control once scapular neutral position is obtained. Surface electromyography (EMG) is an excellent tool to train the patient for proprioception and helps to teach submaximal versus a hard, co-contracted muscle contraction. It may take several minutes to learn neuromuscular control of scapular retraction.

2. Low Row Exercise: Low row exercise incorporates the kinetic chain and is performed by placing the hands along the edge of a table and taking a step forward with the contralateral lower extremity. ${ }^{18-20}$ Performing a low row exercise properly was difficult for this patient. This patient reported pain and "cramping" along the posterior lateral scapula near the axilla during the low row exercise and reproduced pre-surgical symptoms. This was attributed to traction stress on the repair from scapular protraction rather than retraction during the activity and facilitation of the latissimus dorsi rather than rhomboids. It is critical while instructing this exercise that the patient can perform scapular retraction initially and prior to the step through action.

3. Opposite Arm Exercise: Since low row was not tolerated, this subject used the concept of cross-education ${ }^{21,22}$ and irradiation. ${ }^{17}$ Resisted bands for pushing and pulling exercises using the uninvolved extremity through the legs and trunk to incorporate the entire kinetic chain were used to indirectly strengthen the scapular retractors ${ }^{17}$ even while wearing the brace. Specific repetitions were not used but the subject stopped when fatigue indicated by the presence of the familiar periscapular burning. Fatigue occurred quickly initially requiring frequent rests. Performing the activities more frequently with few repetitions is recommended rather than aiming toward a higher number despite the pain. In this subject, symptom resolved within 5-20 minutes using ice and external support.

4. Endurance training: Without the support of the brace, the weight of the extremity pulls the arm down with the scapula going into protraction and depression. One way to gradually wean from the brace is to remove the hand and forearm support from the sling but leave the abductor support strapped around the trunk to allow functional activities with the distal arm at waist level until fatigue symptoms present again. Loosening the anterior strap on the support bolster decreases the percentage of support under the el-

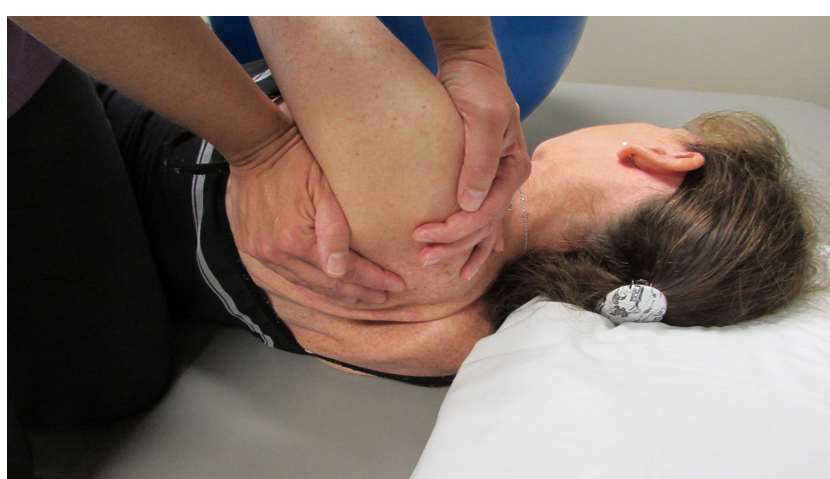

Figure 3: Passive Scapular retraction with elevation

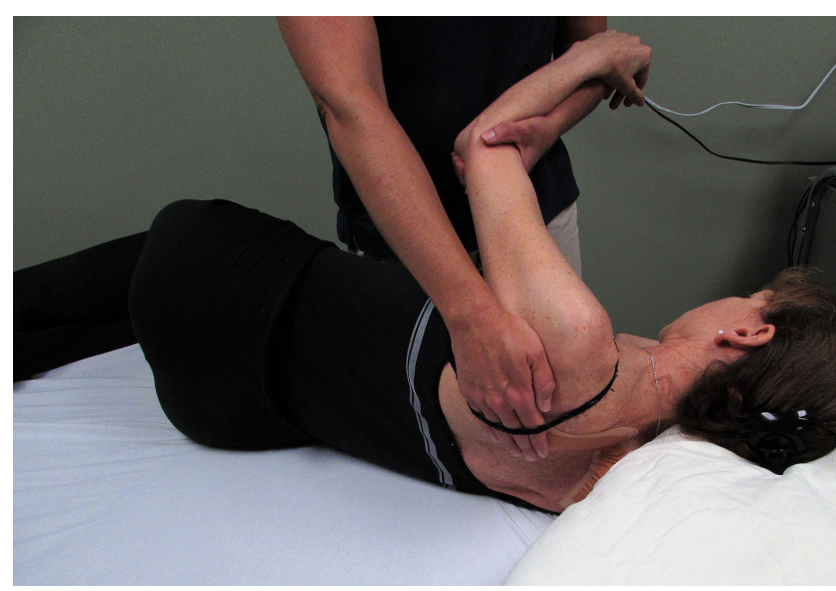

Figure 4: Sidelying soft tissue mobilization to posterior shoulder and lateral scapular musculature

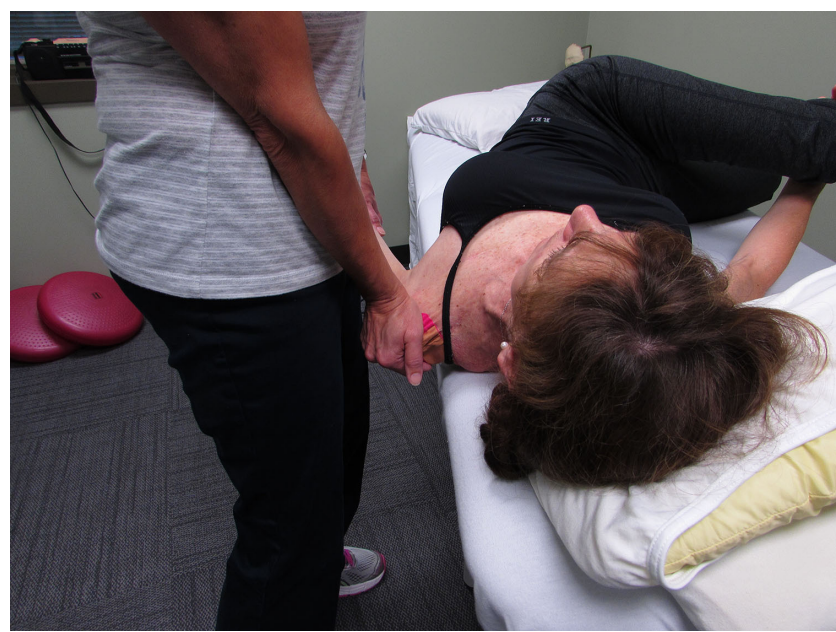

Figure 5: Pectoralis stretching with scapular stabilization and lateral trunk rotation

bow and is easily modified. Tolerance for increased amount of time without support improves gradually. External support for the extremity on a stable or with a pillow allows for functional use of the hand and wrist without the demands of the arm weight. 
PHASE 3: (8-10 WEEKS)

The emphasis is on good mechanics for passive motion in additional planes. Maximal elevation ranges remain limited to $90^{0}$.

\section{STRETCHING AND SOFT TISSUE INTERVENTIONS}

1. Side-lying pectoralis minor stretch: Once the shoulder can reach $70^{0}$ abduction the patient can perform self pectoralis stretching in side-lying using the uninvolved arm to assist with raising the involved extremity up toward the ceiling. Side-lying on a couch with the trunk against the back of the couch provides the scapula and extremity additional support and relieves the scapular muscle "cramping" sensation. (Figure 6)

A key component for this self-stretch is to assure the scapular is fully retracted and parallel to the spine. The therapist can facilitate this position is by placing one hand along the posterior scapula with their fingers on the patient's thoracic spine for feedback and passively raise the arm into abduction with the elbow extended. Allow gravity and the weight of the arm to push the scapula into retraction next to the spine. A second maneuver to facilitate scapular retraction is for the therapist to apply a gentle axial compression force through the wrist for gentle contract (into isometric protraction) and relax (into retraction). With the scapula fully retracted, add slight humeral extension to help posteriorly tilt the scapula followed by elevation for pectoralis minor stretch. If there is pain in the anterior shoulder joint rather than the muscle belly, then the scapula is not fully retracted. Cue the patient to gently shrug or rotate the scapula posteriorly to "adjust it". This stretch exercise should be performed 2-3x/day and held for one to two minutes. It may be performed longer or more frequently and is incorporated prior to the strengthening and motor control activities below.

\section{STRENGTHENING INTERVENTIONS}

1. Small isolated active internal and external rotation: Isolated humeral rotation while in a closed kinetic chain environment decreases co-contraction. With the scapula retracted to neutral and the hand resting softly on a table, cue the patient to gently swing the elbow for full humeral external and internal rotation. Alternatively, slight weight bearing through the extremity next to the hips in sitting facilitates the scapular stabilizers and makes separating the two motions (scapular stability and humeral rotation) easier. Early stages of rehabilitation for strengthening the scapular muscles focuses on neuromotor control to improve proprioception and normalize resting position. ${ }^{10,23}$ The patient should be cued to initiate all passive and active arm motion with the scapular retractors to promote proximal stabilization and train proper scapular mechanics. ${ }^{20}$ Arm abduction exercises are progressed from towel slides and ball rolls to wall slides, so the weight of the arm is supported.

2. Closed kinetic chain weight bearing: Standing with the arms on a table or counter and leaning on them to facilitate scapular retraction can be progressed by shifting weight from side to side. A scale under the arm is a good way

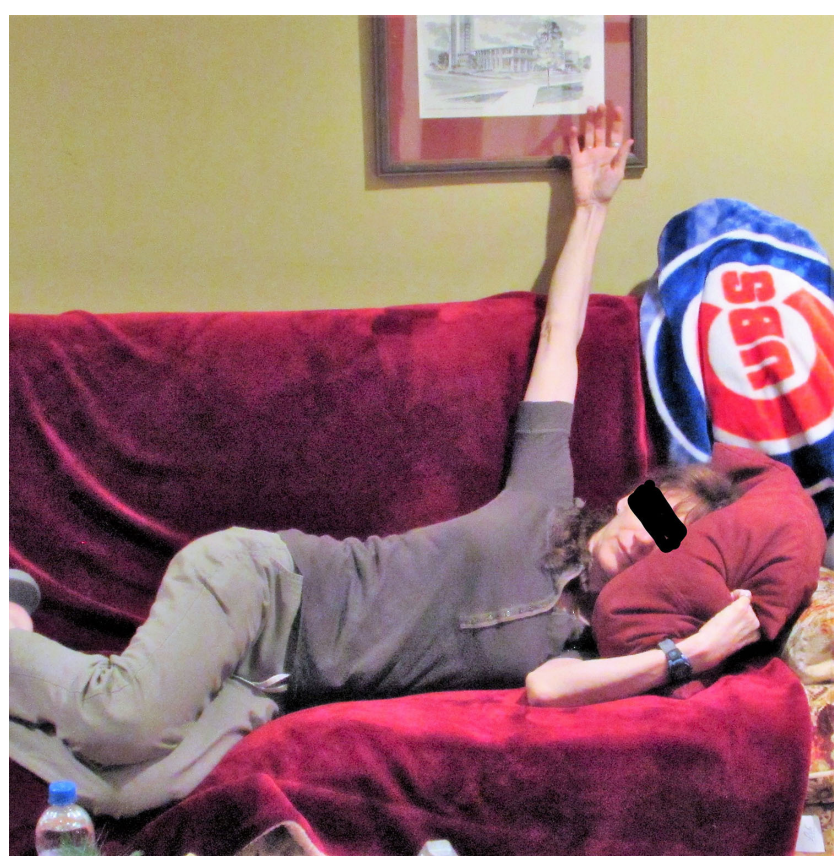

Figure 6: Sidelying pectoralis minor stretch with no weight

to measure tolerable loads and monitor progression of strengthening. ${ }^{24}$ Weight bearing progression can be progressed from two arms to one arm and from standing leaning on a table to prone and quadruped positions adding more support requirements by lifting the opposite extremity or a lower extremity.

\section{PHASE 4: (10-14 WEEKS);}

1. Endurance assistance: Endurance of the scapular muscle to hold the weight of the arm out of the sling improves from minutes to hours during this phase and symptoms of fatigue are easily relieved with less than full support. A pillow or arm resting onto a table will suffice for extremity support. A novel, yet effective technique to be able to offload the weight of the extremity yet allow mobility while walking is to place a small (6") piece of "pool noodle" or towel high under the armpit (Figure 7). It can be held in place with an elastic band through the center of the noodle and around the chest. This serves as a convenient way to wean out of the sling yet give some graded support to the extremity without limiting hand and elbow functions for driving and prolonged walking or working.

\section{5+ WEEKS POST-OPERATIVE}

The Lexington Clinic protocol does not go beyond 14 weeks, but the general guidelines were applied for this subject. Restoration of full glenohumeral rotation mobility without increasing passive stretch on the scapulothoracic joint remained a challenge for up to 6 months. The frequency of one-on-one physical therapy sessions decreased, and subject responsibility increased as the range progressed. In general, the subject was seen $2 \mathrm{x}$ /week from post-op week 6-10 and decreased to weekly for post-operative weeks 
11-16. After four months, seeing the subject every 2-3 weeks for progression of the home program and less frequently from 16-24 weeks would be reasonable. At six months post-operative the subject should have nearly full passive range of motion. Formal visits were dependent on the subject's ability to understand the normal progression of strengthening and mobility.

For this subject, the pain resolved dramatically immediately after surgery though a small amount persisted through the first year. The function and endurance begin to improve most markedly after range was restored by the six months post-operatively time frame but was noticeable month to month at 4, 5, and 6 months. Function continued to improve through the first year and beyond. Pain diminished as the endurance and strength improved over the course of the year. ASES function improved to 42/50 and 47/ 50 levels at one-year post-operative for the left and right arm, respectively.

\section{DISCUSSION}

Efficient upper extremity motion requires a stable base for functional arm and hand mobility; thus, proximal stability for distal mobility. ${ }^{17}$ The scapulothoracic is more proximal and considered the base for the glenohumeral joint and extremity. However, the scapulothoracic joint is predominantly a soft tissue joint. Injuries, such as a muscle rupture, affect the stability and can result in alterations in arm function. ${ }^{9,12}$ As such, when one muscle is ruptured, the antagonists are unopposed which compromises the muscle balance across the joint and alters the kinematics and function for activities such as pushing, pulling, lifting, and reaching. $3,7,12,13$

These alterations in scapular thoracic stability and function, called scapular dyskinesis, have traditionally been considered to be due to neurological problems (long thoracic, accessory or dorsal scapular nerve palsies) or from muscle inhibition secondary to glenohumeral intra-articular disease which may require surgery. $5,8,9,18$ Non-surgical treatments for scapular dyskinesis are usually exercises to help with motor control (ie, timing) or muscle strength at the scapulothoracic and trunk. ${ }^{1-4,6,20}$ Muscle tears, though common in the rotator cuff, are usually not part of the thought paradigm for scapulothoracic dyskinesis. This may explain, in part, why scapular muscle tears are not identified for several years after injury. ${ }^{3}$

For this subject, success in rehabilitation was attributed to five specific interventions added to the Lexington protocol. First, having the therapist take the scapula and manually push it into retraction and elevation toward a position provided pain relief and served as a "starting" point for all other treatments. Without good passive mobility of the scapula, attempts to actively move the scapula through full range were impossible to perform without compensations.

The second intervention, stretching the tight tissues, follows easily from the first. Mobility deficits can be due to tissue tightness, decreased tone, or imbalances of tone with antagonistic muscles. ${ }^{23}$ The tight pectoralis and latissimus dorsi muscles protract and anteriorly tilt the scapula which created tension on the repair and symptoms. Stretching these anterior and short muscles daily was necessary

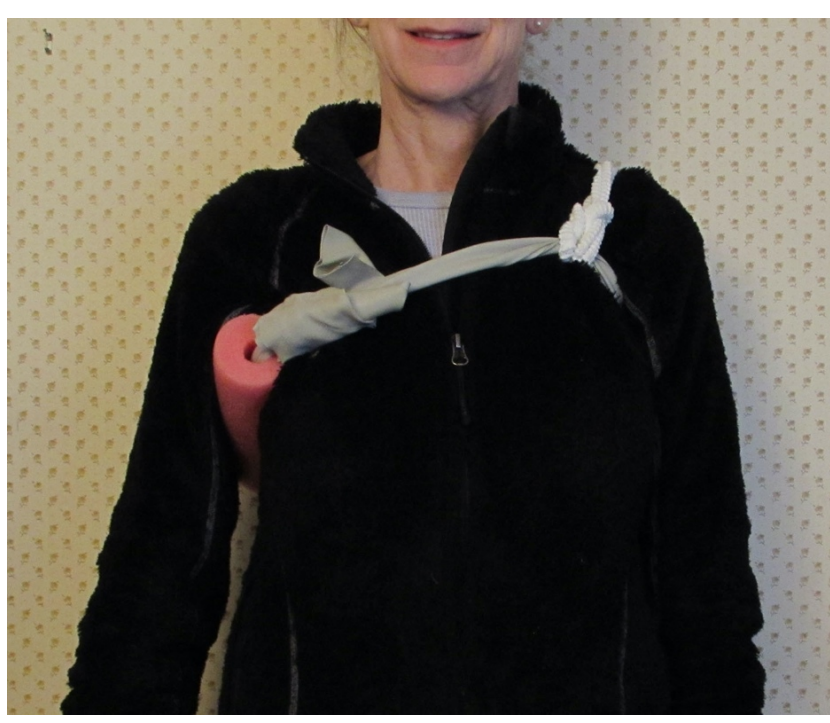

Figure 7: Arm support with pool noodle and TheraBand

to relieve symptoms and critical before any strengthening could occur, including isometrics.

The third intervention in the early stages of rehabilitation that decreased pain and improved the ability for proper kinematics with early stretches was the manual massage techniques to the lateral aspect of the infraspinatus and posterior humeral muscles. A fascial sheath extends from the medial aspect of the rhomboid and is continuous from the anterior surface of the rhomboid to the serratus magnus. ${ }^{25,26}$ There is also a superficial fascia that extends from the superior aspect of the latissimus dorsi muscle across the infraspinatus across to the spine of the scapula ${ }^{25,26} \mathrm{lim}$ iting mobility of the scapular humeral motion. Stabilizing the scapular in neutral and gently massaging the fascia one layer at a time with small ranges of passive arm motion helped to separate motion at the two joints and prepare for normal kinematics. Without this intervention, the fascial restrictions protracted the scapula which placed tension on the repair. In this case, instrumented soft tissue mobility was not performed.

The scapula provides stability for upper extremity motion and functions mainly in the mid to shortened range. ${ }^{23}$ Retraining the scapular thoracic muscles in a retracted position $d$ not in a protracted position was the fourth key intervention. Using a surface biofeedback device was an effective tool to isolate scapular retraction with a submaximal contraction rather than co-contraction. Surface EMG biofeedback was also helpful to assess the timing of the contraction and identify when fatigue occurred.

Finally, pre-operative education treatments to prepare the subject and household for the first weeks post-operatively were invaluable. This subject did not have any preoperative education or expectations prior to the first surgery. Consequently, basic activities such as dressing and sleeping positions were learned by trial and error with pain being the instructor following the first surgery. For the second surgery, knowing how to perform these tasks without increasing stress on the repair made the initial post-opera- 
tive weeks easier, relatively pain free, and increased compliance with the post-operative limitations to limit tension on the repair site.

Since the goal in the first six weeks post-operatively was to allow the repaired muscles to heal without formal therapy or oversight, the subject was solely responsible for assuring that the activities performed were helpful and not harmful to the repair. The pre-operative treatments may also be used to identify and address tight muscles around the shoulder girdle in as a means of getting a "head start" on the post-operative phase. Additionally, limitations along the kinetic chain that prevents activities such as sitting in an upright posture can be addressed. By adding comprehensive pre-operative treatments, the associated impairments were minimized to provide for improved compliance and decreased pain.

In rotator cuff repair, the patients who exhibit poor compliance with post-operative restrictions in the first six weeks showed a relative risk of re-tear and nonhealing 152 times higher than that of a compliant patient. ${ }^{16}$ The unique demands and function of the scapular muscles place the patient at great risk of non-compliance in the initial postoperative phase where there is no formal therapy and the patient is unsupervised. This risk can be mitigated by providing a few pre-operative education sessions and empowering the patient so they can share responsibility for rehabilitation decisions. ${ }^{16}$

\section{CONCLUSION}

Protocols for rotator cuff rehabilitation are not applicable in dealing with scapular muscular reattachment due to the scapular muscles' role in arm elevation. This case report shares a unique perspective as the subject went through this surgery on each arm and is a physical therapist. This program is meant to supplement the Lexington Clinic protocol and provide treating therapist with specific interventions that were key to reduce symptoms in this subject with preoperative program being a unique addition.

Formal therapy begins at approximately 6 weeks with passive shoulder elevation limited to $90^{\circ}$ for approximately 3 months. Full passive motion was re-established by 6 months with active range of motion lagging behind by $20^{\circ}$. Post-operatively, surgical pain resolved in about 2 weeks but the previous 5 years of symptoms such as arm heaviness, headache, referred pain was gone immediately after surgery. Function and strength took about 1 year to return to near normal levels with occasional symptoms of burning due to fatigue that still can recur on occasions.

\section{CONFLICTS OF INTEREST}

No authors have a conflict of interest related to this manuscript.

Submitted: March 25, 2020 CDT, Accepted: December 10, 2020

CDT 


\section{REFERENCES}

1. Cools AM, Ellenbecker TS, Michener LA.

Rehabilitation of scapular dyskinesis. In: Kibler WB, Rivett DA, eds. Disorders of the Scapula and Their Role in Shoulder Injury. Cham, Switzerland: Springer International Publishing; 2017:179-192. https://doi.o rg/10.1007/978-3-319-53584-5_16.

2. Kibler WB, Ludewig PM, Mcclure PW, Michener LA, Bak K, Sciascia AD. Clinical implications of scapular dyskinesis in shoulder injury: The 2013 consensus statement from the 'scapular summit.' Br J Sports Med. 2013;47(14):877-885. doi:10.1136/bjsports-201 3-092425

3. Kibler WB, Sciascia A, Uhl TL. Medial scapular muscle detachment: Clinical presentation and surgical treatment. J Shoulder Elb Surg.

2014;23(1):58-67. doi:10.1016/i.jse.2013.05.008

4. Ludewig PM, Reynolds JF. The association of scapular kinematics and glenohumeral joint pathologies. J Orthop Sports Phys Ther. 2009;39(2):90-104. doi:10.2519/jospt.2009.2808

5. Martin RM, Fish DE. Scapular winging: Anatomical review, diagnosis, and treatments. Curr Rev Musculoskelet Med. 2008;1(1):1-11. doi:10.1007/s1217 8-007-9000-5

6. Roche SJ, Funk L, Sciascia A, Kibler WB. Scapular dyskinesis: The surgeon's perspective. J Shoulder and Elb Surg. 2015;7(4):289-297. doi:10.1177/1758573215 $\underline{595949}$

7. Reuther KE, Thomas SJ, Tucker JJ, et al. Scapular dyskinesis is detrimental to shoulder tendon properties and joint mechanics in a rat model. $J$ Orthop Res. 2014;32(11):1436-1443. doi:10.1002/jor.2 $\underline{2693}$

8. Akgun K, Aktas I, Terzi Y. Winged scapula caused by a dorsal scapular nerve lesion: A case report. Arch Phys Med Rehabil. 2008;89(10):2017-2020. doi:10.101 6/i.apmr.2008.03.015

9. Benedetti MG, Zati A, Stagni SB, Fusaro I, Monesi $\mathrm{R}$, Rotini R. Winged scapula caused by rhomboid paralysis: A case report. Joints. 2016;4(4):247-249. $\underline{\text { do }}$ $\mathrm{i}: 10.11138 / \mathrm{its} / 2016.4 .4 .247$

10. Sciascia AD, Cromwell R, Uhl TL. Rehabilitation for complex scapular dysfunction: Considerations of pain and altered motor patterns. In: Kibler WB, Rivett DA, eds. Disorders of the Scapula and Their Role in Shoulder Injury. Cham, Switzerland: Springer International Publishing; 2017:193-213. https://doi.o rg/10.1007/978-3-319-53584-5_17.
11. Cools AMJ, Struyf F, De Mey K, Maenhout A, Castelein B, Cagnie B. Rehabilitation of scapular dyskinesis: From the office worker to the elite overhead athlete. Br J Sports Med. 2014;48(8):692-697. doi:10.1136/bjsports-2013-092148

12. Kibler WB, Sciascia AD. Disorders of the Scapula and Their Role in Shoulder Injury. Cham, Switzerland: Springer International Publishing AG; 2017.

13. Kibler WB, Sciascia AD. Scapular dyskinesis and glenohumeral instability. In: Kibler WB, Rivett DA, eds. Disorders of the Scapula and Their Role in Shoulder Injury. Cham, Switzerland: Springer International Publishing; 2017:79-89. https://doi.org/10.1007/97 8-3-319-53584-5 7 .

14. Kendall FP, McCreary EK, Provance PG, Rodgers MM. Muscles Testing and Function with Posture and Pain. 5th ed. Baltimore: Lippincott Williams \& Wilkins; 2005.

15. Michener LA, McClure PW, Sennett BJ. American shoulder and elbow surgeons standardized shoulder assessment form, patient self-report section: Reliability, validity, and responsiveness. J Shoulder and Elb Surg. 2002;11(6):587-594. doi:10.1067/mse.20 $\underline{02.127096}$

16. Thigpen CA, Shaffer MA, Gaunt BW, Leggin BG, Williams GR, Wilcox RBI. The American Society of Shoulder and Elbow Therapists' consensus statement on rehabilitation following arthroscopic rotator cuff repair. J Shoulder Elbow Surg. 2016;25(4):521-535. do $\mathrm{i}: 10.1016 / \mathrm{i} . j \mathrm{je} .2015 .12 .018$

17. Adler SS, Beckers D, Buck M, et al. PNF in Practice an Illustrated Guide. Heidelberg Germany: Springer Publishing; 2008.

18. Kibler WB, McMullen I, Uhl TL. Shoulder rehabilitation strategies, guidelines, and practice. $O p$ Techn Sports Med. 2000;8(4):258-267.

19. McMullen J, Uhl TL. A kinetic chain approach for shoulder rehabilitation. J Athl Train.

2000;35(3):329-337.

20. Sciascia A, Cromwell R. Kinetic chain rehabilitation: A theoretical framework. Rehabil Res Pract. 2012;2012:1-9. doi:10.1155/2012/853037

21. Gabriel DA, Kamen G, Frost G. Neural adaptations to resistive exercise: Mechanisms and recommendations for training practices. Sports Med. 2006;36(2):133-149. 
22. Moritani T, deVries HA. Neural factors versus hypertrophy in the time course of muscle strength gain. Am J Phys Med. 1979;58(3):115-130.

23. Sullivan PE, Markos PD, Minor MA. An Integrated Approach to Therapeutic Exercise: Theory and Clinical Application. Reston, Virginia: Reston Publishing Company; 1982.

24. Uhl TL, Carver TJ, Mattacola CG, Mair SD, Nitz AJ. Shoulder musculature activation during upper extremity weight-bearing exercise. J Orthop Sports Phys Ther. 2003;33(3):109-117. doi:10.2519/jospt.200 3.33.3.109
25. Latarjet M, Juttin P. An anatomical and surgical study of the extra-thoracic fascia. Thorax. 1953;8(4):282-287. doi:10.1136/thx.8.4.282

26. Moccia D, Nackashi AA, Schilling R, Ward PJ. Fascial bundles of the infraspinatus fascia: Anatomy, function, and clinical considerations. J Anat. 2016;228(1):176-183. doi:10.1111/joa.12386 


\section{SUPPLEMENTARY MATERIALS}

\section{Appendix A}

Download: https://ijspt.scholasticahq.com/article/21240-post-operative-rehabilitation-for-scapular-musclereattachment-a-case-report/attachment/54022.docx

\section{Appendix B}

Download: https://ijspt.scholasticahq.com/article/21240-post-operative-rehabilitation-for-scapular-musclereattachment-a-case-report/attachment/54543.docx 